\title{
ON A CLASS OF INTEGRAL EQUATIONS WITH DISCONTINUOUS KERNELS*
}

\author{
BY \\ RUDOLPH E. LANGER AND ELEANOR P. BROWN
}

\section{Chapter 1. Introduction}

This paper is given to the discussion of the integral equation in which the kernel $K(x, \xi)$ is continuous, but possesses first partial derivatives which are discontinuous along the line $\xi=x$. It is closely related to a previous paper $\dagger$ in which the case of a discontinuous kernel has been treated. The motivation of the problem and the origin of the work is there discussed. Suffice it to remark here that the present paper originated in a thesis prepared under Birkhoff and presented at Radcliffe College in 1921.+

The integral equation with a kernel having the properties of discontinuity mentioned above is also the subject of a recent paper by A. Hammerstein.§ $\mathrm{He}$ considers, however, only the case in which the kernel is also symmetric. Assuming the existence of infinitely many positive characteristic values he obtains an asymptotic form for the characteristic functions.

The present paper, without the hypothesis of symmetry, establishes the existence and derives the asymptotic forms of the characteristic values, and gives for the characteristic functions an asymptotic expression which is considerably more explicit than that obtained by Hammerstein for a more restricted case. The closure of the set of solutions is discussed and the problem of the expansion of an arbitrary function in a series of solutions is treated. The methods used are on the whole the same as those used in the earlier paper referred to above, and where the procedure is similar it has been passed over briefly here. One improvement, however, which is also applicable to the case there treated may be mentioned, namely, the proof that the set of characteristic functions is closed has been materially simplified. This was accomplished by the use of an extension of Birkhoff's theorem on

* Presented to the Society, December 28, 1926; received by the editors December 1, 1926.

$\dagger$ Langer, these Transactions, vol. 28 (1926). This paper will be referred to in the text simply as paper 1.

$\ddagger$ By Eleanor Pairman, now Mrs. Eleanor P. Brown.

\& Über die asymptotische Darstellung der Eigenfunktionen linearer Integralgleichungen, Mathematische Annalen, 1924, p. 113. Cf. also his paper in Mathematische Annalen, 1926, p. 102, where an analogous situation for the integral equation in two variables is treated. 
closure, and of a general theorem on the closure of the solutions of an integral equation.*

The final chapter outlines the theory of an equation which does not satisfy the general hypotheses made, and shows that under such circumstanoes the solutions may differ radically in character from those of the cases more fully treated.

Chapter 2. The normalization of the equation

1. The type of equation treated. The integral equation to be discussed is of the form

$$
y(t)+\lambda^{2} \int_{\alpha}^{\beta} \Gamma(t, \tau) y(\tau) d \tau=0,
$$

where $\lambda$ is a complex parameter, while the kernel $\Gamma(t, \tau)$ is real and satisfies the following hypotheses:

(i) $\Gamma(t, \tau)$ is continuous in the region

$$
\bar{R}\left\{\begin{array}{l}
\alpha \leqq t \leqq \beta \\
\alpha \leqq \tau \leqq \beta,
\end{array}\right.
$$

and possesses partial derivatives of order 2 which are continuous in the open regions

$$
\bar{R}^{\prime}\left\{\begin{array} { l } 
{ \alpha \leqq \tau < t } \\
{ \alpha \leqq t \leqq \beta , }
\end{array} \quad \overline { R } ^ { \prime \prime } \left\{\begin{array}{l}
t<\tau \leqq \beta \\
\alpha \leqq t \leqq \beta,
\end{array}\right.\right.
$$

and approach finite limiting values on the boundary $\tau=t$.

$$
\left.\Gamma_{t}(t, \tau)\right]_{\tau=t-}^{\tau=t+}=\phi(t) \neq 0 .
$$

(iii) The functions

$$
\left.\frac{\partial^{2} \Gamma(t, \tau)}{\partial t^{2-j} \partial \tau^{j}}\right]_{\tau=t-}^{\tau=t+} \quad(j=0,1,2)
$$

each possess a continuous derivative on the interval $(\alpha, \beta)$.

* Langer, Three theorems on closure of biorthogonal systems of functions, Bulletin of the American Mathematical Society, vol. 33 (1927), pp. 97-105. We note here that in this paper the requirement that the solutions of the equation numbered 8 form a biorthogonal set should have appeared as part of the hypothesis. 
2. The change of variables. Under the hypotheses made the function $\phi(t)$ possesses a continuous derivative of second order. ${ }^{*}$ There is no loss of generality moreover in assuming $\phi(t)>0$. The quantities

$$
x=\int_{\alpha}^{t}\{\phi(t)\}^{1 / 2} d t, \quad \xi=\int_{\alpha}^{\tau}\{\phi(\tau)\}^{1 / 2} d \tau
$$

may serve, therefore, as new independent variables. In terms of them equation (1) takes the form

$$
\bar{y}(x)=\lambda^{2} \int_{a}^{b} \Omega(x, \xi) \bar{y}(\xi) d \xi,
$$

where

and

$$
\bar{y}(x) \equiv y(t), \quad a=0, \quad b=\int_{\alpha}^{\beta}\{\phi(\tau)\}^{1 / 2} d \tau,
$$

$$
\Omega(x, \xi) \equiv-\frac{\Gamma(t, \tau)}{\{\phi(\tau)\}^{1 / 2}} \cdot
$$

The kernel $\Omega(x, \xi)$ of this equation is continuous, while

$$
\left.\Omega_{x}(x, \xi)\right]_{\xi-x-}^{\xi-x+} \equiv-1
$$

Let the function $\psi(x)$ be defined now by the relation

$$
\left.\Omega_{x x}(x, \xi)\right]_{\xi-x-}^{\xi-x+}=\psi(x) .
$$

Then with the introduction of

$$
u(x)=\bar{y}(x) e^{(1 / 2) \int_{a}^{x} \psi(x) d x}
$$

as a new dependent variable the equation (3) in turn takes the form

$$
u(x)=\lambda^{2} \int_{a}^{b} Q(x, \xi) u(\xi) d \xi,
$$

where

$$
Q(x, \xi)=\Omega(x, \xi) e^{(1 / 2) \int_{\xi}^{x} \psi(x) d x} .
$$

It is to be observed that

$$
\left.Q_{x x}(x, \xi)\right]_{\xi-x-}^{\xi-x+} \equiv 0
$$

* Cf., e.g., paper $1, \S 2$. 
3. The equation of normal form.* The kernel $Q(x, \xi)$ may possibly be orthogonal on the interval $(a, b)$ to certain continuous functions either of $x$ or of $\xi$, and if this is the case either equation (5) or its associated equation will fail to satisfy certain hypotheses which must subsequently be made. We shall make, then, the following hypothesis concerning the given equation, namely:

(iv) The kernel $Q(x, \xi)$ is orthogonal to at most a finite number $r$ of linearly independent functions $\psi_{i}(x)$ or to at most a finite number $s$ of linearly independent functions $\phi_{i}(\xi)$, these functions being possessed of continuous derivatives of order 2 . If $r \neq 0, s \neq 0$, then with proper assignment of subscripts the system of functions

$$
\left\{\phi_{i}(x), \psi_{i}(x)\right\} \quad(i=1,2, \cdots, p)
$$

may be made biorthogonal and normal, $\uparrow$ where $p$ is the lesser of the two integers $r$ and $s$, or $p=r=s$.

Under this hypothesis we may suppose that

$$
\int_{a}^{b} \phi_{i}(x) \psi_{i}(x) d x=\delta_{i j} \quad(i, j=1,2, \cdots, p),
$$

where $\delta_{i j}$ is zero or one according as $i \neq j$ or $i=j$.

Consider now the integral equation

$$
u(x)=\lambda^{2} \int_{a}^{b} K(x, \xi) u(\xi) d \xi,
$$

where

$$
K(x, \xi)=Q(x, \xi)+\sum_{i=1}^{p} \phi_{i}(x) \psi_{i}(\xi) .
$$

If we assume the kernel of this equation to be orthogonal on $(a, b)$ to a continuous function $\sigma(x)$, it follows that

$$
\int_{a}^{b} Q(x, \xi) \sigma(x) d x+\sum_{i=1}^{p} \psi_{i}(\xi) \int_{a}^{b} \phi_{i}(x) \sigma(x) d x=0 .
$$

Multiplying this equation by $\phi_{k}(x)$ and integrating we find because of (6) that

$$
\int_{a}^{b} \phi_{k}(x) \sigma(x) d x=0 \quad(k=1,2, \cdots, p),
$$

* This step in the normalization was not made in paper 1 , but is equally applicable to the problem there treated.

† Cf. Goursat, Cours d'A nalyse, vol. 3, p. 392. 
and hence because of the preceding equation that $\sigma(x)$ must be orthogonal also to $Q(x, \xi)$. Accordingly $\sigma(x)$ must be a linear combination of the functions $\psi_{i}(x)$ and since it is orthogonal to $\phi_{k}(x)$ it follows that if $p=r$ then $\sigma \equiv 0$. Similarly it can be shown that if $K(x, \xi)$ is orthogonal to a continuous function $\omega(\xi)$, then $\omega \equiv 0$ if $p=s$. It will be recalled that either $p=r$, or $p=s$, or both.

It is clear that the features of discontinuity of the kernel $Q(x, \xi)$ are preserved in the kernel $K(x, \xi)$. If the definitions of the partial derivatives of $K(x, \xi)$ are completed by assigning to them on the line $\xi=x$ their limiting values as $\xi \rightarrow x, \xi<x$ the equation (7) will be said to represent the normal form. Its distinguishing characteristics are the following:

(A) There exists either no function of $x$ or no function of $\xi$ which is continuous with its second derivative, is orthogonal to $K(x, \xi)$, and is not identically zero.

(B) $K(x, \xi)$ is continuous in the region

$$
R\left\{\begin{array}{l}
a \leqq x \leqq b \\
a \leqq \xi \leqq b,
\end{array}\right.
$$

and possesses partial derivatives to those of order $n \geqq 2$ which are continuous in the regions

$$
R_{1}\left\{\begin{array} { l } 
{ a \leqq \xi \leqq x } \\
{ a \leqq x \leqq b , }
\end{array} \text { and } \quad R _ { 2 } \left\{\begin{array}{l}
x<\xi \leqq b \\
a \leqq x \leqq b
\end{array}\right.\right.
$$

and in the latter region approach finite limiting values at each point of the boundary $\xi=x$.

$$
\left.\left.K_{x}(x, \xi)\right]_{\xi-x}^{\xi-x+} \equiv-1, \quad K_{\xi}(x, \xi)\right]_{\xi-x}^{\xi-x+} \equiv 1 .
$$

$$
\left.\left.\left.K_{x x}(x, \xi)\right]_{\xi-x}^{\xi-x+} \equiv K_{x \xi}(x, \xi)\right]_{\xi-x}^{\xi-x+} \equiv K_{\xi \xi}(x, \xi)\right]_{\xi-x}^{\xi-x+} \equiv 0 .
$$

4. The relation between the given equation and the normal equation. Inasmuch as the functions $Q(x, \xi)$ and $\sum_{i=1}^{p} \phi_{i}(x) \psi_{i}(\xi)$ are orthogonal, the sets of characteristic values and functions of (7) are composed respectively of the corresponding sets for the two equations, namely (5), and the equation with kernel $\sum_{i=1}^{p} \phi_{i}(x) \psi_{i}(\xi)$. $^{*}$

* Goursat, Cours d'A nalyse, vol. 3, p. 402. 
This latter equation is found, however, to admit of the single characteristic value $\lambda=1$, with $\phi_{1}(x), \cdots, \phi_{p}(x)$ constituting a complete set of characteristic functions.

THEOREM 1. Every integral equation of type (1) whose kernel possesses the properties (i), (ii), (iii), (iv) may be associated with an equation of normal form as defined above.

Chapter 3. Transformation of the integral equation into AN INTEGRO-DIFFERENTIAL SYSTEM

5. The auxiliary differential system. We shall make the hypothesis that for the normal integral equation in hand the number $n$ under property (B), §3, satisfies the relation

$$
n \geqq 4 \text {. }
$$

Then we consider in conjunction with this equation the differential system

$$
\begin{aligned}
& y^{\prime \prime}(x)=0, \\
& L_{i}(y)=\mu_{i 1} y(a)+\mu_{i 2} y^{\prime}(a)+\nu_{i 1} y(b)+\nu_{i 2} y^{\prime}(b)=0 \quad(i=1,2),
\end{aligned}
$$

and its adjoint system

$$
\begin{aligned}
& z^{\prime \prime}(x)=0, \\
& M_{i}(z)=\bar{\mu}_{i 1} z(a)+\bar{\mu}_{i 2} z^{\prime}(a)+\bar{\nu}_{i 1} z(b)+\bar{\nu}_{i 2} z^{\prime}(b)=0 .
\end{aligned}
$$

The coefficients $\mu_{i j}, \nu_{i j}$ will be regarded as parameters to be chosen arbitrarily subject to the restriction that they shall not satisfy certain relations (finite in number) which arise in the deductions. Thus to begin with

(9) $\mu_{i j}, \nu_{i j}$ shall be chosen so that system (8) is incompatible.

This restriction insures the existence of the Green's function $G(x, \xi)$. If we designate by $L_{i}(\omega)$, when $\omega=\omega(x, \xi)$ is any function of two variables, the the result produced by the operation $L_{i}$ on $\omega$ considered as a function of $x$, and by $M_{i}(\omega)$ the result produced by $M_{i}$ on $\omega$ as a function of $\xi$, then with proper definitions for the partial derivatives of $G(x, \xi)$ on the line $\xi=x$ we have

I. $G(x, \xi)$ is continuous in $R$ and its partial derivatives are continuous in $R_{1}$ and $R_{\mathbf{2}}$.

II.

$$
\left.\left.G_{x}(x, \xi)\right]_{\xi=x}^{\xi=x+} \equiv-1, \quad G_{\xi}(x, \xi)\right]_{\xi-x}^{\xi=x+} \equiv 1
$$


III. $\left.\quad G_{x x}(x, \xi) \equiv G_{\xi \xi}(x, \xi) \equiv 0, \quad G_{x \xi}(x, \xi)\right]_{\xi=x}^{\xi-x+} \equiv 0$.

IV. $\quad L_{i}(G)=M_{i}(G) \equiv 0$.

6. The function $K(x, \xi)$. We abbreviate by setting

$$
W_{i}(x)=M_{i}(K), \quad V_{i}(\xi)=L_{i}(K)
$$$$
(i=1,2) \text {. }
$$

These functions are continuous together with their first $(n-1)$ derivatives on the interval $(a, b)$. Moreover

$$
L_{i}\left(W_{j}\right)=M_{j}\left(V_{i}\right),
$$

for it is found by direct substitution and the use of properties (C) and (D) of the kernel $K(x, \xi)$ that (11) is equivalent to

$$
\mu_{j 1} \bar{\mu}_{i 2}-\mu_{j 2} \bar{\mu}_{i 1}-\nu_{j 1} \bar{\nu}_{i 2}+\nu_{j 2} \bar{\nu}_{i 1}=0
$$$$
(i, j=1,2) \text {, }
$$

while the validity of this relation may be established as follows. The operators $M_{j}$ and $L_{i}$ are connected by the relation*

$$
\int_{a}^{b}\left[z y^{\prime \prime}-y z^{\prime \prime}\right] d x=\sum_{i=1}^{4} L_{i}(y) M_{5-i}(z),
$$

where $L_{i}(y)$ for $i=3,4$ may be considered as given by formulas (8) with any determination of coefficients $\mu_{i j}, \nu_{i j}, i=3,4, j=1,2$, for which the determinant

$$
\theta=\left|\mu_{i 1}, \mu_{i 2}, \nu_{i 1}, \nu_{i 2}\right| \quad(i=1,2,3,4)
$$

does not vanish. By direct integration of the left member of (12) its value is found to be

$$
-y^{\prime}(a) z(a)+y(a) z^{\prime}(a)+y(b) z(b)-y(b) z^{\prime}(b) .
$$

Solving equations

$$
L_{i}=\mu_{i 1} y(a)+\mu_{i 2} y^{\prime}(a)+\nu_{i 1} y(b)+\nu_{i 2} y^{\prime}(b) \quad(i=1,2,3,4),
$$

we obtain the expressions

$$
y(a)=y_{1}, \quad y^{\prime}(a)=y_{2}, \quad y(b)=y_{3}, \quad y^{\prime}(b)=y_{4},
$$

where

$$
y_{i}=\frac{1}{\theta} \sum_{j=1}^{4} C_{j i} L_{j}(y)
$$

*Cf. Birkhoff, Boundary value and expansion problems, these Transactions, vol. 9 (1908), p. 375 . 
the constant $C_{j i}$ being the cofactor of the element in the $j$ th row and $i$ th column of $\theta$. If these values are substituted in (12a), we have both members of (12) expressed as linear forms in $L_{i}(y)$. Equating the coefficients of $L_{i}(y)$ for $i=4,3$ we obtain the forms $M_{j}(z), j=1,2$. As a result we find that we may cho ose

$$
\bar{\mu}_{i 1}=C_{5-i, 2}, \quad \bar{\mu}_{i 2}=-C_{5-i, 1}, \quad \bar{\nu}_{i 1}=-C_{5-i, 4}, \quad \bar{\nu}_{i 2}=C_{5-i, 3} .
$$

With these values it is evident that (11a) is an identity.

Let the quantity $\Delta$ be defined by the relation

$$
\Delta=\left|\begin{array}{ll}
L_{1}\left(W_{1}\right) & L_{1}\left(W_{2}\right) \\
L_{2}\left(W_{1}\right) & L_{2}\left(W_{2}\right)
\end{array}\right|
$$

Inasmuch as the constants ${ }^{-} \bar{\mu}_{i j}, \bar{\nu}_{i j}$ can be expressed as polynomials in $\mu_{i j}$, $\nu_{i j}$, the same is true of $\Delta$. We shall make the following hypothesis concerning the given equation, namely that

$$
\Delta \not \equiv 0 \text { in } \mu_{i j}, \nu_{i j} .
$$

Then we may suppose these parameters chosen so that

$$
\Delta \neq 0 \text {. }
$$

The function $K(x, \xi)$ is defined now by the formula

$$
K(x, \xi)=\frac{1}{\Delta}\left|\begin{array}{lll}
K(x, \xi) & W_{1}(x) & W_{2}(x) \\
V_{1}(\xi) & L_{1}\left(W_{1}\right) & L_{1}\left(W_{2}\right) \\
V_{2}(\xi) & L_{2}\left(W_{1}\right) & L_{2}\left(W_{2}\right)
\end{array}\right|
$$

With the use of relation (11) it is readily verified that $K(x, \xi)$ shares with $K(x, \xi)$ its characteristics (B) to (D), while

$$
L_{\mathbf{i}}(\mathrm{K}) \equiv M_{\boldsymbol{i}}(\mathrm{K}) \equiv 0
$$$$
(i=1,2) \text {. }
$$

7. The relation between $K(x, \xi)$ and $G(x, \xi)$. The function

$$
\omega(x, \xi)=K(x, \xi)-G(x, \xi)
$$

is continuous together with its partial derivatives of second order in $R$, and satisfies the differential system

$$
\begin{aligned}
\frac{\partial^{2} \omega(x, \xi)}{\partial^{2} \xi} & =K_{\xi \xi}(x, \xi), \\
M_{i}(\omega) & \equiv 0 \quad(i=1,2) .
\end{aligned}
$$

It follows* that we have for $G(x, \xi)$ as a function of $x$ the integral equation

* Cf. paper 1, 87 . 


$$
K(x, \xi)-G(x, \xi)=-\int_{a}^{b} E(x, t) G(t, \xi) d t,
$$

where

$$
E(x, t)=-K_{t t}(x, t)
$$

From (15) we may write

$$
K(x, t)=K(x, t)-\theta_{1}(x) V_{1}(t)-\theta_{2}(x) V_{2}(t),
$$

where we observe in passing that

$$
L_{i}\left(\theta_{j}\right)=\delta_{i j} \quad(i, j=1,2) .
$$

From (18) it is seen that the parameters $\mu_{i j}, \nu_{i j}$ are contained in the kernel of (17) only rationally and only in such terms as are products of a function of $x$ by a function of $t$. It can be shown because of this that the Fredholm determinant $D$ and the first minor $D(x, t)$ of the kernel $E(x, t)$ are themselves rational functions of these parameters.* We shall make the hypothesis that for the given equation

$$
D \not \equiv 0 \text { in } \mu_{i j}, \nu_{i j},
$$

and shall suppose the parameters chosen so that

$$
D \neq 0 \text {. }
$$

Then the reciprocal $F(x, t)$ of the kernel $E(x, t)$ exists and the relation (17) may be written in the "solved" form

$$
G(x, t)=K(x, t)-\int_{a}^{b} F(x, t) K(t, \xi) d t .
$$

8. Properties of $F(x, t)$. The kernel $E(x, t)$ and hence also the reciprocal are continuous in $R$. From (16) we obtain the relation

$$
L_{i}(E) \equiv 0,
$$

and from Fredholm's identities

$$
E(x, \xi)+F(x, \xi)=\int_{a}^{b} E(x, t) F(t, \xi) d t=\int_{a}^{b} F(x, t) E(t, \xi) d t
$$

it follows further that

$$
L_{i}(F) \equiv 0
$$

* Cf. the method of proof in paper 1, $\$ 8$ or Bateman, H., A formula for the solving function of a certain integral equation of the second kind, Messenger of Mathematics, vol. 37(1907), p. 179. 
and that $F_{x}(x, \xi)$ is possessed of partial derivatives to those of order $(n-3)$ which are continuous in $R_{1}$ and $R_{2}$. Lastly since $F(x, \xi)=D(x, \xi) / D$, this reciprocal is rational in the parameters $\mu_{i j}, \nu_{i j}$.

9. Transformation of equation (7). Let $u(x)$ now be any solution of the normalized equation (7). Th. 2n it follows from (7), (10) and (18) that

$$
\lambda^{2} \int_{a}^{b} V_{i}(\xi) u(\xi) d \xi=L_{i}(u)
$$

and

$$
\lambda^{2} \int_{a}^{b} K(x, \xi) u(\xi) d \xi=u(x)-L_{1}(u) \theta_{1}(x)-L_{2}(u) \theta_{2}(x) .
$$

With these relations we find upon multiplying (21) by $\lambda^{2} u(\xi)$ and integrating that

(25) $\lambda^{2} \int_{a}^{b} G(x, \xi) u(\xi) d \xi=u(x)-L_{1}(u) \Phi_{1}(x)-L_{2}(u) \Phi_{2}(x)-\int_{a}^{b} F(x, t) u(t) d t$ where

$$
\Phi_{i}(x)=\theta_{i}(x)-\int_{a}^{b} F(x, t) \theta_{i}(t) d t \quad(i=1,2) .
$$

We observe that $\Phi_{i}(x)$ possesses continuous derivatives to those of order $(n-2)$ and that

$$
L_{i}\left(\Phi_{j}\right)=\delta_{i j} \quad(i, j=1,2) .
$$

Returning to relation (25) and differentiating it twice weobtain an integrodifferential equation satisfied by $u(x)$. If we abbreviate by setting

$$
\begin{aligned}
& \Phi_{i}{ }^{\prime}(x)=\phi_{i}(x), \\
& \left.F_{x}(x, \xi)\right]_{\xi=x}^{\xi=x+}=g(x), \\
& F_{x x}(x, \xi)=f(x, \xi),
\end{aligned}
$$

and associate the equation last obtained with (24) above the result may be formulated as in the following theorem.

THEOREM 2. If the kernel of the normalized integral equation is such that the conditions (v), (vi), and (vii) are satisfied then every solution of the integral equation is also a solution of the integro-differential system

(a) $u^{\prime \prime}(x)-\lambda^{2} u(x)=L_{1}(u) \phi_{1}(x)+L_{2}(u) \phi_{2}(x)+\int_{a}^{b} f(x, \xi) u(\xi) d \xi-g(x) u(x)$,

$$
L_{i}(u)=\lambda^{2} \int_{a}^{b} V_{i}(\xi) u(\xi) d \xi \quad(i=1,2) .
$$


Of the functions $g(x)$ and $\phi_{i}(x)$, the first possesses continuous derivatives to order $(n-3)$ while the others possess continuous derivatives to order $(n-4)$. The function $f(x, \xi)$ and its partial derivatives to order $(n-4)$ are continuous in the regions $R_{1}$ and $R_{2}$. i.e.

10. The associated integral equation. The equation associated with (7),

$$
v(x)=\lambda^{2} \int_{a}^{b} K(\xi, x) v(\xi) d \xi,
$$

is also in normal form if the partial derivatives of the kernel are properly defined on the line $\xi=x$. The deductions already made are easily adapted, therefore, to apply to this equation as well. Thus we may set $\bar{K}(x, \xi)$ $=K(\xi, x)$, and let the two sets of parameters $\mu_{i j}, \nu_{i j}$ and $\bar{\mu}_{i j}, \bar{\nu}_{i j}$ be interchanged. If we distinguish the various functions arising then in the consideration of (7) by superscribing them with a bar, it is readily found that

$$
\bar{W}_{i}(x) \equiv V_{i}(x), \quad \bar{V}_{i}(\xi) \equiv W_{i}(\xi),
$$

and, since $L_{i}$ and $M_{i}$ must be interchanged, that $\bar{\Delta}=\Delta$. The condition $\bar{\Delta} \not \equiv 0$ in the parameters is therefore already covered. It is, however, necessary to make the further hypothesis that

$$
\bar{D} \not \equiv 0 \text { in } \bar{\mu}_{i j}, \bar{\nu}_{i j}
$$

The conclusions embodied in Theorem 2 for equation (7) can then be drawn in an analogous manner for equation $(\overline{7})$.

The substitution of the equation (5) for equation (7) in $\$ 3$ can now be motivated. If the kernel $K(x, \xi)$ is orthogonal on $(a, b)$ to a function $\sigma(x)$ it follows from formulas (15) and (10) that

$$
\int_{a}^{b} K(x, \xi) \sigma(x) d x=0
$$

identically in $\mu_{i j}, \nu_{i j}$. But differentiating this equation twice it follows that

$$
\sigma(\xi)=\int_{a}^{b} E(x, \xi) \sigma(x) d x,
$$

and hence that $D \equiv 0$ in $\mu_{i j}, \nu_{i j}$. Similarly if $K(x, \xi)$ is orthogonal to $\omega(\xi)$, then $\bar{D} \equiv 0$. If the kernel of equation (5) is orthogonal both to functions of $x$ and to functions of $\xi$, therefore, a discussion similar to that above but applied to the equation (5) or to its associated equation would necessarily be stopped by conditions (vii) and (vii). 
11. The transformation of system (29). The equivalence of system (29) and equation (7) will be established if it is shown that every solution of the former solves also the latter. Let us suppose the system (29) given. Then the functions $\Phi_{i}(x), i=1,2$, and $F(x, \xi)$ may be determined respectively as the unique continuous solutions of the differential systems

$$
\begin{aligned}
& y^{\prime \prime}(x)=\phi_{i}(x), \\
& L_{j}(y)=\delta_{j i}
\end{aligned}
$$$$
(i, j=1,2),
$$

and as the unique solution continuous in $R_{1}$ and $R_{2}$ of the system

$$
\begin{gathered}
\frac{\partial^{2} Y(x, \xi)}{\partial x^{2}}=f(x, \xi), \\
\left.L_{i}(Y) \equiv 0, \quad Y_{x}(x, \xi)\right]_{\xi-x}^{\xi-x+}=g(x) .
\end{gathered}
$$

Let $\sigma(x)$ be constructed now by the formula

$$
\begin{gathered}
\sigma(x)=u(x)-\lambda^{2} \int_{a}^{b} G(x, \xi) u(\xi) d \xi-L_{1}(u) \Phi_{1}(x)-L_{2}(u) \Phi_{2}(x) \\
-\int_{a}^{b} F(x, t) u(t) d t,
\end{gathered}
$$

$u(x)$ being any solution of (29a). By (29a), IV, (22b), and (27), this function is a solution of the incompatible system (8). Hence it follows that $\sigma(x) \equiv 0$, namely that $u(x)$ is a solution of (25).

The function $E(x, \xi)$ is determined as the reciprocal of $F(x, \xi)$, and the functions $K(x, \xi)$ and $\theta_{i}(x)$ are respectively determined by relations (17) and (26).

Let (17) be multiplied by $\lambda^{2} u(\xi)$ and integrated with respect to $\xi$. Eliminating

$$
\lambda^{2} \int_{a}^{b} G(x, \xi) u(\xi) d \xi
$$

from the result by means of (25), we find because of (26) and (23) that every solution of $(29 a)$ is also a solution of the equation

$$
u(x)=\lambda^{2} \int_{a}^{b} K(x, \xi) u(\xi) d \xi+L_{1}(u) \theta_{1}(x)+L_{2}(u) \theta_{2}(x) .
$$

If the function $u(x)$ is a solution of the entire system (29) we may substitute further for $L_{i}(u)$ in (30) the values given by (29b). Because of (18) this results in reducing equation (30) to the form (7). 
THEOREM 3. Under the hypotheses of Theorem 2 every solution of the integrodifferential system (29) is also a solution of the normalized integral equation (7).

12. The general system of type (29). In the preceding section we were concerned with the system (29) which was deduced from equation (7). This fact affected the deductions only to the extent that the function $E(x, \xi)$ reciprocal to $F(x, \xi)$ was known to exist. In any case the final function is found from (21), (26) and (18) to satisfy the relation

$$
K(x, \xi)=\left\{G(x, \xi)-V_{1}(\xi) \Phi_{1}(x)-V_{2}(\xi) \Phi_{2}(x)\right\}+\int_{a}^{b} F(x, t) K(t, \xi) d t
$$

which may be solved if $F(x, t)$ possesses a reciprocal. Hence we may state

Theorem 4. If the function $F(x, \xi)$ associated in the manner above with an integro-differential system of type (29) possesses a reciprocal, then the system is equivalent to an integral equation of the second kind.

Clearly the equivalence of equation $(\overline{7})$ with the integro-differential system deduced from it may be established similarly under the hypotheses made.

\section{Chapter 4. The existence of a solution CF the INTEGRO-DIFFERENTIAL EQUATION}

13. Notation and lemmas. We shall adopt now the following as generic symbols:

$N$ to represent a positive constant sufficiently large;

$H(x, \xi, \lambda)$ to represent a function which is uniformly bounded for $|\lambda|>N$;

$\epsilon(x, \xi, \lambda)$ to represent a function which approaches zero uniformly as $|\lambda| \rightarrow \infty$;

$B(x, \xi, \lambda)$ to represent a function which for $|\lambda|>N$ is uniformly bounded and in integrable as to $\xi$ uniformly in $x$ and $\lambda$;

$E(x, \lambda)$ to represent a function which is uniformly bounded for $|\lambda|>N$, and which is continuous in $x$ and analytic in $\lambda$;

$M$ to represent a constant which is positive or zero.

It is convenient also to have at hand the following lemmas. $\dagger$ In them $\sigma$ denotes the real part of $\lambda$.

* Cf. Langer and Tamarkin, $A$ notion of uniform integrability, Bulletin of the American Mathematical Society, vol. 32 (1926), p. 335.

$\dagger$ For proofs see paper $1, \$ 17$. 
LEMMA 1. If $\sigma \leqq M$ and $a \leqq z \leqq b$, then

$$
\int_{a}^{z} e^{\lambda(z-\xi)} B(x, \xi, \lambda) d \xi=\epsilon(x, z, \lambda) .
$$

LEMMA 2. If $\sigma \geqq-M$ and $a \leqq z \leqq b$, then

$$
\int_{b}^{z} e^{\lambda(z-\xi)} B(x, \xi, \lambda) d \xi=\epsilon(x, z, \lambda) .
$$

14. Transformation of the integro-differential equation. Let $P(u, x)$ designate the right hand member of equation (29a) and consider the equation

$$
u^{\prime \prime}(x)-\lambda^{2} u(x)=P(u, x)+E(x, \lambda) \text {. }
$$

Looking upon it as a differential equation we may write

$$
u(x)=c_{1} e^{\lambda x}+c_{2} e^{-\lambda x}+\frac{1}{2 \lambda} \int_{*}^{x}\left[e^{\lambda(x-\xi)}-e^{-\lambda(x-\xi)}\right][P(u, \xi)+E(\xi, \lambda)] d \xi,
$$

the coefficients $c_{1}$ and $c_{2}$ being arbitrary but constant with respect to $x$, and * representing a constant which may be chosen arbitrarily for each term of the integrand. Confining the attention to the case $\sigma \leqq M$ we assume for the moment that equation (32a) is possessed of a solution for the following values of the arbitrary elements:

$$
\begin{aligned}
& c_{1}=k e^{-\lambda a} \\
& c_{2}=0,
\end{aligned}
$$

( $k$ a constant),

* $=a$ for terms of the integrand involving $e^{\lambda(x-\xi)}$,

$*=b$ for terms of the integrand involving $e^{-\lambda(x-\xi)}$.

With the abbreviations

$$
\begin{aligned}
\bar{\phi}_{i}(x) & =\frac{1}{2} \int_{a}^{b} e^{\lambda|x-\xi|} \phi_{i}(\xi) d \xi \\
\bar{E}(x) & =\frac{1}{2} \int_{a}^{b} e^{\lambda|x-\xi|} E(x, \xi) d \xi \\
\bar{f}(x, \xi) & =\frac{1}{2}\left\{\int_{a}^{b} f(t, \xi) e^{\lambda|x-t|} d t-g(\xi) e^{\lambda|x-\xi|}\right\},
\end{aligned}
$$

the equation may then be written

$$
\begin{gathered}
u(x)=k e^{\lambda(x-a)}+\frac{1}{\lambda}\left\{L_{1}(u) \bar{\phi}_{1}(x)+L_{2}(u) \bar{\phi}_{2}(x)+\bar{E}(x)\right. \\
\left.+\int_{a}^{b} \bar{f}(x, \xi) u(\xi) d \xi\right\} .
\end{gathered}
$$


Looking upon this as an explicit evaluation of $u(x)$ and forming from it the expressions $L_{i}(u)$, we obtain

$$
L_{i}(u)=L_{1}(u) \Phi_{i 1}+L_{2}(u) \Phi_{i 2}+T_{i}+\int_{a}^{b} R_{i}(\xi) u(\xi) d \xi \quad(i=1,2),
$$

where

$$
\begin{gathered}
\Phi_{i j}=c_{i 1} \bar{\phi}_{j}(a)+c_{i 2} \bar{\phi}_{j}(b), \quad c_{i 1}=\frac{\mu_{i 1}}{\lambda}-\mu_{i 2}, \\
R_{i}(\xi)=c_{i 1} \bar{f}(a, \xi)+c_{i 2} \bar{f}(b, \xi), \quad c_{i 2}=\frac{\nu_{i 1}}{\lambda}+\nu_{i 2}, \\
T_{i}=\left(\mu_{i 1}+\lambda \mu_{i 2}\right) k+\lambda c_{i 2} k e^{\lambda(b-a)}+c_{i 1} \bar{E}(a)+c_{i 2} \bar{E}(b) .
\end{gathered}
$$

The equations (33) compose an algebraic system for the quantities $L_{i}(u)$ with determinant

$$
C=\left|\begin{array}{rr}
1-\Phi_{11} & -\Phi_{12} \\
-\Phi_{21} & 1-\Phi_{22}
\end{array}\right|
$$

By Lemmas 1 and $2, \bar{\phi}_{i}(x)=\epsilon(x, \lambda)$, and hence $\Phi_{i j}=\epsilon(\lambda)$ and $C^{-1}=H(\lambda)$. Hence the system (33) may be solved if $|\lambda|>N$. If we denote by $\gamma_{i j}$ the minor of the element in the $i$ th row and $j$ th column of $C$, the solution is

$$
L_{i}(u)=C^{-1} \sum_{j=1}^{2} \gamma_{j i}\left[T_{j}+\int_{a}^{b} R_{i}(\xi) u(\xi) d \xi\right] .
$$

With these values equation (32b) becomes

$$
u(x)=\theta(x, \lambda)+\int_{a}^{b} \Omega(x, \xi, \lambda) u(\xi) d \xi,
$$

where

$$
\theta(x, \lambda)=k e^{\lambda(x-a)}+\frac{\bar{E}(x)}{\lambda}+\frac{C^{-1}}{\lambda} \sum_{i, j=1}^{2} \bar{\phi}_{i}(x) \gamma_{j i} T_{i}
$$

and

$$
\Omega(x, \xi, \lambda)=\frac{1}{\lambda}\left\{\bar{f}(x, \xi)+C^{-1} \sum_{i, j=1}^{2} \bar{\phi}_{i}(x) \gamma_{j i} R_{j}(\xi)\right\} .
$$

15. The existence of a solution of equation (32). Returning now to the equation (32) let the functions $\theta(x, \lambda)$ and $\Omega(x, \xi, \lambda)$ be constructed from its 
coefficients by the formulas above. It is readily found then that for $\sigma \leqq M$ and $|\lambda|>N, \theta(x, \lambda), \theta_{x}(x, \lambda) / \lambda, \Omega_{x}(x, \xi, \lambda) / \lambda$ are bounded, while

$$
|\Omega(x, \xi, \lambda)|<\frac{1}{2(b-a)} .
$$

Hence the equation (32c) possesses a solution $u(x)$, which satisfies the relations $|u(x)|<A,\left|u^{\prime}(x) / \lambda\right|<A^{\prime}$, and which is analytic in $\lambda$ for $|\lambda|>N$. To show that $u(x)$ satisfies also equation (32) it is necessary only to reverse the deductions above.

The case $\sigma \geqq-M$ may be treated similarly to that above, the arbitrary elements in (32a) being chosen as follows:

$$
\begin{aligned}
& c_{1}=0, \quad c_{2}=k e^{\lambda a}, \\
& *=b \text { for terms involving } e^{\lambda(x-\xi)}, \\
& *=a \text { for terms involving } e^{-\lambda(x-\xi)}
\end{aligned}
$$

The results also are similar to those there obtained.

If the function $E(x, \lambda)$ vanishes identically the equation considered coincides with (29a) and we may summarize our results as follows.

THEOREM 5. If the parameter $\lambda$ is restricted to any region bounded by a line parallel to the axis of imaginaries and exterior to a circle sufficiently large with center at $\lambda=0$ then the equation (29a) admits of a solution $u(x)$ which is analytic in $\lambda$ and satisfies the relations

$$
|u(x)|<A(\text { a constant }), \quad\left|\frac{u^{\prime}(x)}{\lambda}\right|<A^{\prime}(\text { a constant }) .
$$

If $E(x, \lambda) \not \equiv 0$, the choice $k=0$ is possible. If we assume further that $E(x, \lambda)$ is integrable in $x$ uniformly with respect to $\lambda$, then by Lemmas 1 and $2, \bar{E}(x)=\epsilon(x, \lambda)$, and with $k=0, \theta(x, \lambda)=\epsilon(x, \lambda) / \lambda$. In this case we have the

THEOREM 6. If the parameter $\lambda$ is confined to a region as described in Theorem 5 and if the function $E(x, \lambda) \neq 0$ is integrable in $x$ uniformly with respect to $\lambda$, then equation (32) admits of a solution $u(x)$ which is analytic in $\lambda$ and which is such that

$$
u(x)=\frac{\epsilon(x, \lambda)}{\lambda}, \quad u^{\prime}(x)=\epsilon(x, \lambda)
$$


Chapter 5. The solution of the integro-differential EQUATION

16. The probable form of a solution. To derive the probable form of a solution of equation (29a) we consider the scheme of successive approximation defined by the formula

$$
u_{j}^{\prime \prime}(x)-\lambda^{2} u_{j}(x)=P\left(u_{j-1}, x\right),
$$

where $P(u, x)$ is the right hand member of (29a). A particular function $u_{i}(x)$ in terms of $u_{j-1}(x)$ is given by

$$
u_{i}(x)=e^{\lambda x}+\frac{1}{2} \int_{*}^{x}\left[e^{\lambda(x-t)}-e^{-\lambda(x-t)}\right] P\left(u_{j-1}, t\right) d t,
$$

with any constant choice of $*$ for each term of the integrand. The initial function $u_{0}(x) \equiv 0$ leads to $u_{1}(x)=e^{\lambda x}$. With the use of Lemmas 1 and 2 and with proper choice of $*$ from the values $*=a, *=b$ and $*=\frac{1}{2}(a+b)$ it is found that for $j=2$, (36) admits of a solution of the form

$$
u_{2}(x)=e^{\lambda a} H(x, \lambda)+e^{\lambda b} H(x, \lambda)+e^{\lambda x} H(x, \lambda)+e^{\lambda(b+a-x)} H(x, \lambda) .
$$

In the further repetition of the process the introduction of terms of a type not already represented is avoidable. We are thus led to expect the existence of a solution with the functional form of $u_{2}(x)$ above.

17. The formal determination of coefficients. We shall write the functions $H(x, \lambda)$ now as series in descending powers of $\lambda$, and seek to evaluate the coefficients. With the notation

we have then

$$
[\omega(x)]=\sum_{j=0}^{\infty} \frac{\omega_{j}(x)}{\lambda^{j}}
$$

$$
u(x)=e^{\lambda x}[\gamma(x)]+e^{\lambda(b+a-x)}[\delta(x)]+e^{\lambda b}[\beta(x)]+e^{\lambda a}[\alpha(x)] .
$$

Substituting this into equation (29a) and integrating by parts the terms involving $f(x, \xi)[\gamma(\xi)]$ and $f(x, \xi)[\delta(\xi)]$ we obtain the result

$$
\begin{aligned}
& e^{\lambda x}\left\{2 \lambda\left[\gamma^{\prime}(x)\right]+\left[\gamma^{\prime \prime}(x)\right]+g(x)[\gamma(x)]\right. \\
& \left.\left.\quad-\sum_{i=0}^{\infty} \frac{1}{\lambda^{i+1}} \sum_{i=0}^{i}(-1)^{i} \frac{\partial}{\partial \xi^{j}}\left[f(x, \xi) \gamma_{i-j}(\xi)\right]\right]_{\xi-x}^{\xi-x+}\right\} \\
& +e^{\lambda(b+a-x)}\{\}+e^{\lambda b}\{\}+e^{\lambda a}\{\}=0 .
\end{aligned}
$$


Equating to zero the coefficient of $e^{\lambda x} / \lambda^{l}$ for $l=-1,0,1,2, \cdots$, we obtain equations from which the quantities $\gamma_{i}(x)$ may be successively determined. In particular the choice $\gamma_{0}=1$ is possible. Further equating to zero the coefficient of $e^{\lambda(b+a-x)} / \lambda^{l}$ we find as a possible choice $\delta_{i}(x) \equiv 0$ for all $i$, while the terms in $e^{\lambda b} / \lambda^{l}$ and $e^{\lambda a} / \lambda^{l}$ similarly yield formulas for the determination of the quantities $\beta_{i}(x)$ and $\alpha_{i}(x)$. With the values already chosen we find in particular $\beta_{0}=\alpha_{0}=0$.

18. The function $\bar{u}(x)$. The extent to which the formal process thus developed is actually applicable is limited by the number of derivatives which the coefficients of equation (29a) admit. Referring to $\$ 9$, therefore, and considering the formulas obtained, we find that $\gamma_{i}(x)$ possesses $(n-1-i)$ derivatives while $\beta_{2 i-1}(x), \beta_{2 i}(x), \alpha_{2 i-1}(x), \alpha_{2 i}(x)$ possess derivatives of order $(n-2-2 i)$. The last of these coefficients which may be differentiated twice are therefore $\gamma_{n-3}(x), \beta_{n-4}(x), \alpha_{n-4}(x)$ if $n$ is even and $\gamma_{n-3}(x), \beta_{n-5}(x), \alpha_{n-5}(x)$, if $n$ is odd. To avoid duplication in the discussion we shall suppose that for the case in hand $n$ is even. Then the function

$$
\begin{aligned}
\bar{u}(x)=e^{\lambda x} & \left\{1+\frac{\gamma_{1}(x)}{\lambda}+\cdots+\frac{\gamma_{n-3}(x)}{\lambda^{n-3}}\right\} \\
& +e^{\lambda b}\left\{\frac{\beta_{1}(x)}{\lambda}+\cdots+\frac{\beta_{n-4}(x)}{\lambda^{n-4}}\right\} \\
& +e^{\lambda a}\left\{\frac{\alpha_{1}(x)}{\lambda}+\cdots+\frac{\alpha_{n-4}(x)}{\lambda^{n-4}}\right\}
\end{aligned}
$$

is twice differentiable. It is readily seen to satisfy an equation of the form

$$
\bar{u}^{\prime \prime}(x)-\lambda^{2} \bar{u}(x)=P(\bar{u}, x)+\frac{1}{\lambda^{n-5}}\left\{e^{\lambda b} B(x, \lambda)+e^{\lambda a} B(x, \lambda)\right\},
$$

the functions $B(x, \lambda)$ being analytic in $\lambda$ for $|\lambda|>N$.

19. The true solutions. If $u(x)$ is any solution of equation (29a) the function $u(x)-\bar{u}(x)$ is a solution of equation (39) and conversely. On the other hand if equation (39) is multiplied by $\lambda^{n-5} e^{-\lambda a}$ it takes, for $\sigma \leqq M$, the form (32) with

$$
E(x, \lambda)=e^{\lambda(b-a)} B(x, \lambda)+B(x, \lambda) .
$$

This function $E(x, \lambda)$ is integrable in $x$ uniformly with respect to $\lambda$. It follows by Theorem 6 that this modified equation possesses a solution of the form $\epsilon(x, \lambda) / \lambda$, with derivatives of the form $\epsilon(x, \lambda)$. Hence we conclude that for $\sigma \leqq M$ equation (29a) possesses a solution given by 


$$
\begin{gathered}
u(x)-\tilde{u}(x)=\frac{\epsilon(x, \lambda) e^{\lambda a}}{\lambda^{n-4}} \\
u^{\prime}(x)-\bar{u}^{\prime}(x)=\frac{\epsilon(x, \lambda) e^{\lambda a}}{\lambda^{n-5}} .
\end{gathered}
$$

For $\sigma \geqq-M$ a similar argument may be applied to equation (39) multiplied by $\lambda^{n-5} e^{-\lambda b}$. Hence we conclude that equation (29a) possesses for $\sigma \geqq-M$ a solution given by

$$
\begin{aligned}
u(x)-\bar{u}(x) & =\frac{\epsilon(x, \lambda) e^{\lambda b}}{\lambda^{n-4}}, \\
u^{\prime}(x)-\bar{u}^{\prime}(x) & =\frac{\epsilon(x, \lambda) e^{\lambda b}}{\lambda^{n-5}} .
\end{aligned}
$$

It is clear that we may summarize these results as follows.

THEOREM 7. If $\lambda$ is confined to a region of the type described in Theorem 5 then the integro-differential equation (29a) admits of a solution $u_{1}(x)$ of the form

$$
\begin{aligned}
u_{1}(x)=e^{\lambda x} & \left\{1+\frac{\gamma_{1}(x)}{\lambda}+\cdots+\frac{\gamma_{n-4}(x)}{\lambda^{n-4}}\right\} \\
& +e^{\lambda b}\left\{\frac{\beta_{1}(x)}{\lambda}+\cdots+\frac{\beta_{n-4}(x)+\epsilon(x, \lambda)}{\lambda^{n-4}}\right\} \\
& +e^{\lambda a}\left\{\frac{\alpha_{1}(x)}{\lambda}+\cdots+\frac{\alpha_{n-4}(x)+\epsilon(x, \lambda)}{\lambda^{n-4}}\right\},
\end{aligned}
$$

the first derivatives of the functions $\epsilon(x, \lambda)$ involved being of the form $\lambda \epsilon(x, \lambda)$.

The equation (29a) involves $\lambda$ only to the even power two. It follows that we have a second solution of this equation, namely

$$
u_{2}(x, \lambda)=u_{1}(x,-\lambda)
$$

Clearly these two solutions are linearly independent.

20. The associated equation. Inasmuch as this chapter and the preceding one involve no new hypotheses concerning the equation (7), it is clear that a precisely analogous discussion of the associated equation $(\overline{7})$ is possible. The corresponding integro-differential equation admits, therefore, of solutions $v_{1}(x), v_{2}(x)$ whose form may be obtained from $u_{1}(x)$ and $u_{2}(x)$ by replacing $\gamma_{i}, \beta_{i}$ and $\alpha_{i}$, by $\bar{\gamma}_{i}, \bar{\beta}_{i}$ and $\bar{\alpha}_{i}$ respectively.

\section{Chapter 6. The characteristic values}

21. The characteristic equation. Since the equation (29a) is linear we have as a solution the expression 


$$
u(x)=c_{1} u_{1}(x)+c_{2} u_{2}(x),
$$

where $c_{1}$ and $c_{2}$ are arbitrary constants. By Theorems 2 and 3 , such a solution will satisfy the given integral equation if and only if it satisfies also the equations (29b). Setting

$$
\omega_{i j}(\lambda)=L_{i}\left(u_{j}\right)-\lambda^{2} \int_{a}^{b} V_{i}(\xi) u_{j}(\xi) d \xi \quad(i, j=1,2),
$$

and substituting in (29b) the form (42) we obtain as the relations to be satisfied

$$
c_{1} \omega_{i 1}(\lambda)+c_{2} \omega_{i 2}(\lambda)=0 \quad(i=1,2) .
$$

These equations compose a homogeneous system for the constants $c_{1}$ and $c_{2}$ and admit of a solution if

$$
\left|\begin{array}{ll}
\omega_{11}(\lambda) & \omega_{12}(\lambda) \\
\omega_{21}(\lambda) & \omega_{22}(\lambda)
\end{array}\right|=0 .
$$

This equation in $\lambda$ we shall call the characteristic equation. Its roots, which are the values of $\lambda$ for which (42) includes a solution of the integral equation, are the characteristic values.

To deduce the functional form of (45) we substitute for $\lambda^{2} u_{j}(\xi)$ in (43) its value as the second term in (29a). Upon integrating twice by parts the resulting term in $u_{i}^{\prime \prime}(\xi)$ we obtain the formula

$$
\text { (43a) } \omega_{i 1}(\lambda)=k_{i 1} u_{1}(a)+k_{i 2} u_{1}(b)+k_{i 3} u_{1}^{\prime}(a)+k_{i 4} u_{1}^{\prime}(b)-\int_{a}^{b} \Omega_{i}(\xi) u_{1}(\xi) d \xi \text {, }
$$

where

$$
k_{i 4}=\nu_{i 1}-V_{i}(b)+\nu_{12} \int_{a}^{b} V_{i}(\xi) \phi_{1}(\xi) d \xi+\nu_{22} \int_{a}^{b} V_{i}(\xi) \phi_{2}(\xi) d \xi
$$

and $k_{i j}, j=1,2,3$, are given by similar forms, and where

$$
\Omega_{i}(\xi)=V_{i}^{\prime \prime}(\xi)+V_{i}(\xi) g(\xi)-\int_{a}^{b} f(t, \xi) V_{i}(t) d t \quad(i=1,2) .
$$

Substituting in (43a) the forms

$$
\begin{aligned}
u_{1}(x) & =e^{\lambda x}+e^{\lambda} b_{\epsilon}(x, \lambda)+e^{\lambda a_{\epsilon}} \epsilon(x, \lambda), \\
u_{1}^{\prime}(x) & =\lambda\left\{e^{\lambda x}+e^{\lambda} b_{\epsilon}(x, \lambda)+e^{\lambda a_{\epsilon}} \epsilon(x, \lambda)\right\},
\end{aligned}
$$

obtained from Theorem 7 , we find that

$$
\omega_{i 1}(\lambda)=\lambda e^{\lambda a}\left\{k_{i 3}+\epsilon(\lambda)\right\}+\lambda e^{\lambda b}\left\{k_{i 4}+\epsilon(\lambda)\right\} .
$$


For the reductions here involved it is convenient to use the results of the following lemma.

LEMMA $3^{*}$. If $z_{1}$ and $z_{2}$ are any points of the interval $(a, b)$, then

$$
\int_{z_{1}}^{z_{2}} e^{\lambda t} H(\xi, t, \lambda) d t=e^{\lambda z_{z}} H\left(z_{2}, \xi, \lambda\right)+e^{\lambda z_{1}} H\left(z_{1}, \xi, \lambda\right) .
$$

From (41) and (43) it is readily seen that $\omega_{i 2}(\lambda)=\omega_{i 1}(-\lambda)$. Hence (43b) yields for the characteristic equation the form

$$
\left|\begin{array}{ll}
k_{13} & k_{14} \\
k_{23} & k_{24}
\end{array}\right|\left[e^{\lambda(b-a)}-e^{-\lambda(b-a)}\right]+e^{\lambda(b-a)} \epsilon(\lambda)+\epsilon(\lambda)+e^{-\lambda(b-a)} \epsilon(\lambda)=0 .
$$

The quantities $k_{i j}$ are rational functions of the parameters $\mu_{i j}, \nu_{i j}$, as is seen from formula (46) etc. The vanishing or non-vanishing character of the determinant $\left(k_{13} k_{24}-k_{14} k_{23}\right)$ must, however, be independent of the choice of these parameters, since it vitally affects the structure of the characteristic equation, which depends only on the integral equation given. $\dagger$ We shall make the hypothesis that the given integral equation is such that

$$
\left|\begin{array}{ll}
k_{13} & k_{14} \\
k_{23} & k_{24}
\end{array}\right| \neq 0 . \ddagger
$$

The characteristic equation may then be written, for $|\lambda|>N$, in the form

$$
e^{2 \lambda(b-a)}+\epsilon(\lambda) e^{\lambda(b-a)}-[1+\epsilon(\lambda)]=0 .
$$

22. The characteristic values. The equation (45a) is formally a quadratic in $e^{\lambda(b-a)}$. Factoring it we obtain

$$
e^{\lambda(b-a)}=1+\epsilon(\lambda), \quad e^{\lambda(b-a)}=-1+\epsilon(\lambda) .
$$

From this it follows that

$$
\begin{aligned}
& \lambda=\frac{2 m \pi i}{b-a}+\epsilon(\lambda), \\
& \lambda=\frac{(2 m+1) \pi i}{b-a}+\epsilon(\lambda) \quad(m= \pm M, \pm(M+1), \cdots) .
\end{aligned}
$$

It is readily shown $\S$ that for $|m|$ sufficiently large these equations admit respectively just one root in the neighborhood of $2 m \pi i /(b-a)$, and $(2 m+1) \pi i /(b-a)$. These are the characteristic values.

* For the proof of this lemma see paper $1, \$ 23$.

$\dagger$ The analogous situation arising in paper $1, \S 33$, is illustrated in paper 1 , appendix.

$\ddagger$ A case in which this condition is not met is discussed in chapter 10.

\& Cf. paper 1, \$33. 
By the classical theory of integral equations, associated equations have the same characteristic values. We observe, therefore, that our results are obtainable by the methods outlined when either equation (7) or equation $(\overline{7})$ satisfies the hypotheses made. If we designate by $\lambda_{m}$ the characteristic value in the neighborhood of $m \pi i /(b-a)$, we may formulate the result as follows:

THEOREM 8. Under the hypotheses (v), (vi) and (viii) and either (vii) or (vii), the normalized integral equation (7) admits of infinitely many characteristic values. For $|m|$ sufficiently large these values are given by the formula

$$
\lambda_{m}=\frac{m \pi i}{b-a}+\epsilon_{m} \quad(m= \pm M, \pm(M+1), \cdots),
$$

where $\lim _{|m|+\infty} \epsilon_{m}=0$.

23. A more precise formula for $\lambda_{m}$. The deductions just made if carried out with the assumption of a greater number of derivatives of the kernel of the integral equation will lead to a more precise formula. Thus if $n \geqq 6$, then by Theorem 7 there exists a function $u_{1}(x)$ given by the theorem for $n=6$. With this form it is found that

$$
\begin{aligned}
\omega_{i 1}(\lambda) & =\lambda e^{\lambda a}\left\{k_{i 3}+\frac{k_{i 3} \gamma_{1}(a)+k_{i 1}}{\lambda}+\frac{H(\lambda)}{\lambda^{2}}\right\} \\
& +\lambda e^{\lambda b}\left\{k_{i 4}+\frac{k_{i 4} \gamma_{1}(b)+k_{i 2}}{\lambda}+\frac{H(\lambda)}{\lambda^{2}}\right\},
\end{aligned}
$$

and hence that the functions $\epsilon(\lambda)$ in (45a) are of the type $A / \lambda+(\bar{A}+\epsilon(\lambda)) / \lambda^{2}$, where $A$ and $\bar{A}$ are constants. This leads to a formula

$$
\lambda_{2 m}=\frac{2 m \pi i}{b-a}+\frac{A}{2 m}+\frac{\bar{A}+\epsilon_{2 m}}{(2 m)^{2}},
$$

with a similar form with distinct constants for $\lambda_{2 m+1}$. We observe now, however, that since the given integral equation involves $\lambda$ to an even power, $-\lambda_{m}$ is also a characteristic value. Clearly we may conclude from Theorem 8 that for $|m|$ sufficiently large $-\lambda_{m}=\lambda_{-m}$. Hence it follows that in the formula above $\bar{A}=0$. We have, therefore,

THEOREM 9. If, for the integral equation considered in Theorem $8, n \geqq 6$, then the characteristic values are represented by a formula

$$
\lambda_{m}=\frac{m \pi i}{b-a}+\frac{A_{1}+(-1)^{m} A_{2}}{m}+\frac{\epsilon_{m}}{m^{2}},
$$

where $A_{1}$ and $A_{2}$ are constants and $\lim _{|m| \rightarrow \infty} \epsilon_{m}=0$. 


\section{Chapter 7. The characteristic Functions}

24. The solutions of the integral equation. The values $\lambda_{m}$ have been determined so that for suitable choice of the constants $c_{1}$ and $c_{2}$ formula (42) yields a solution of the integral equation. From (44) we find, moreover, because of (45) that

$$
c_{1}: c_{2}=-\left[h_{1} \omega_{12}\left(\lambda_{m}\right)+h_{2} \omega_{22}\left(\lambda_{m}\right)\right]:\left[h_{1} \omega_{11}\left(\lambda_{m}\right)+h_{2} \omega_{21}\left(\lambda_{m}\right)\right],
$$

with any choice of the constant multipliers $h_{1}$ and $h_{2}$. We shall choose these multipliers so that

$$
\begin{aligned}
& h_{1} k_{14}+h_{2} k_{24}=1, \\
& h_{1} k_{13}+h_{2} k_{23}=0,
\end{aligned}
$$

which is possible because of (viii). If we observe then that $e^{\lambda_{m} x}$ is uniformly bounded we find from (49) with the use of (43b) that

$$
c_{1}: c_{2}=\left[e^{-\lambda_{m} b}+\epsilon\left(\lambda_{m}\right)\right]:\left[e^{\lambda_{m} b}+\epsilon\left(\lambda_{m}\right)\right]
$$

and hence from (42) with the use of (40a) that

$$
u\left(x, \lambda_{m}\right)=e^{\lambda_{m}(b-x)}+e^{-\lambda_{m}(b-x)}+\epsilon\left(x, \lambda_{m}\right) .
$$

Substituting for $\lambda_{m}$ its value as given by (47) this reduces to

$$
u\left(x, \lambda_{m}\right)=2 \cos \frac{m \pi(b-x)}{b-a}+\epsilon(x, m) .
$$

25. The associated equation. The characteristic values of the equation (7) being also those of the associated equation ( $\overline{7})$, the considerations of this and the preceding chapter apply equally well to either equation if both satisfy the hypotheses made. Under this assumption we may conclude, therefore, that the equation ( $\overline{7})$ admits of a solution $v\left(x, \lambda_{m}\right)$ of precisely the form of $u\left(x, \lambda_{m}\right)$ above. Integrating the product of these solutions we find that

$$
\int_{a}^{b} u\left(x, \lambda_{m}\right) v\left(x, \lambda_{m}\right) d x=2(b-a)+\epsilon(m) .
$$

The solutions may, therefore, be normalized by multiplying them by

$$
[2(b-a)+\epsilon(m)]^{-1 / 2} .
$$

Since the given equation involves only $\lambda^{2}$ it follows that the solution for $\lambda_{m}$ is identically that for $\lambda_{-m}$. Setting $u_{m}(x)=u\left(x, \lambda_{m}\right)=u\left(x, \lambda_{-m}\right)$, $v_{m}(x)=v\left(x, \lambda_{m}\right)=v\left(x, \lambda_{-m}\right)$, we may summarize our results as follows: 
Theorem 10. Under the hypotheses (v), (vi), (vii), (viii) and (viii) the normalized characteristic functions of the given integral equation and its associated equation are given respectively for $m$ sufficiently large by the formulas

$$
\begin{aligned}
& u_{m}(x)=\left(\frac{2}{b-a}\right)^{1 / 2} \cos \frac{m \pi(b-x)}{b-a}+\epsilon(x, m), \\
& v_{m}(x)=\left(\frac{2}{b-a}\right)^{1 / 2} \cos \frac{m \pi(b-x)}{b-a}+\epsilon(x, m) .
\end{aligned}
$$

26. The more precise formulas. If for the equation in hand $n \geqq 6$, more explicit formulas may be deduced by the method above. Thus we obtain from (48) and (43c)

$$
\begin{aligned}
c_{1}: c_{2}= & {\left[e^{-\lambda_{m} b}-\frac{A e^{-\lambda_{m} b}+\bar{A} e^{-\lambda_{m} a}}{\lambda_{m}}+\frac{H\left(\lambda_{m}\right)}{\lambda_{m}{ }^{2}}\right] } \\
& :\left[e^{\lambda_{m} b}+\frac{A e^{\lambda_{m} b}+\bar{A} e^{\lambda_{m} a}}{\lambda_{m}}+\frac{B\left(\lambda_{m}\right)}{\lambda_{m}{ }^{2}}\right],
\end{aligned}
$$

and using the form of $u_{1}\left(x, \lambda_{m}\right)$ given by Theorem 7 for $n=6$, we obtain

$$
\begin{aligned}
u\left(x, \lambda_{m}\right)= & {\left[e^{\lambda_{m}(b-x)}+e^{-\lambda_{m}(b-x)}\right]+\frac{A-\gamma_{1}(x)}{\lambda_{m}}\left[e^{\lambda_{m}(b-x)}-e^{-\lambda_{m}(b-x)}\right] } \\
& -\frac{\bar{A}}{\lambda_{m}}\left[e^{\lambda_{m}(x-a)}-e^{-\lambda_{m}(x-a)}\right]+\frac{H\left(\lambda_{m}\right)}{\lambda_{m}{ }^{2}} .
\end{aligned}
$$

Substituting in this the value of $\lambda_{m}$ as given by (47a) and observing that

$$
(-1)^{m} \sin \frac{m \pi(b-x)}{b-a}=-\sin \frac{m \pi(x-a)}{b-a},
$$

we obtain the result

$$
\begin{aligned}
u\left(x, \lambda_{m}\right)= & 2 \cos \frac{m \pi(b-x)}{b-a}+\frac{B(x)}{m} \sin \frac{m \pi(b-x)}{b-a} \\
& +\frac{H(x)}{m} \sin \frac{m \pi(x-a)}{b-a}+\frac{H(x, m)}{m^{2}} .
\end{aligned}
$$

Precisely as in $\$ 27$ we conclude that the associated equation admits of a solution which is of the form $u\left(x, \lambda_{m}\right)$ above with distinct functions $H$. Moreover

$$
\int_{a}^{b} u\left(x, \lambda_{m}\right) v\left(x, \lambda_{m}\right) d x=2(b-a)+\frac{H(m)}{m^{2}} .
$$


Hence we have the following theorem.

THEOREM 11. If for the equation considered in Theorem $10, n \geqq 6$, the normalized solutions of the equation and its associated equation are given for $m$ sufficiently large by the formulas

$$
\begin{array}{r}
u_{m}(x)=\left(\frac{2}{b-a}\right)^{1 / 2} \cos \frac{m \pi(b-x)}{b-a}+\frac{1}{m}\left[H_{1}(x) \sin \frac{m \pi(b-x)}{b-a}\right. \\
\left.+H_{2}(x) \sin \frac{m \pi(x-a)}{b-a}\right]+\frac{H(x, m)}{m^{2}}, \\
v_{m}(x)=\left(\frac{2}{b-a}\right)^{1 / 2} \cos \frac{m \pi(b-x)}{b-a}+\frac{1}{m}\left[\bar{H}_{1}(x) \sin \frac{m \pi(b-x)}{b-a}\right. \\
\left.+\bar{H}_{2}(x) \sin \frac{m \pi(x-a)}{b-a}\right]+\frac{H(x, m)}{m^{2}} .
\end{array}
$$

Chapter 8. The closure of the set of characteristic functions

27. Application of a theorem of Birkhoff. We shall assume now that for the given integral equation

$$
n \geqq 6 \text {. }
$$

To show that the sets $u_{m}(x)$ and $v_{m}(x)$ are closed we shall employ the following extension of a theorem of Birkhoff.

Theorem. Let $\left\{u_{m}(x), v_{m}(x)\right\}, m=0,1,2, \cdots$, be a normalized biorthogonal system of continuous functions and $\left\{\bar{u}_{m}(x) \bar{v}_{m}(x)\right\}$ be a second biorthogonal normalized system which is such that (a) the series

$$
\sum_{m=0}^{\infty}\left[u_{m}(x)-\bar{u}_{m}(x)\right] v_{m}(y), \quad a \leqq x, y \leqq b,
$$

converges uniformly to a function $H(x, y)$ less than $1 /(b-a)$ in absolute value, and (b) the convergence is such that the series upon being multiplied by any continuous function may be integrated term by term as to $x$ to yield a uniformly convergent series. Then if the set $\left\{u_{m}(x)\right\}$ involves a $k$-fold lack of closure, the lack of closure of the set $\left\{\bar{u}_{m}(x)\right\}$ is at most $k$-fold.*

28. The integro-differential system with an additional parameter. Consider the system

$$
\begin{aligned}
& u^{\prime \prime}(x)-\lambda^{2} u(x)=\eta P(u, x) \\
& L_{i}(u)=\eta \lambda^{2} \int_{a}^{b} V_{i}(\xi) u(\xi) d \xi \quad(i=1,2) .
\end{aligned}
$$

* Langer, Bulletin of the American Mathematical Society, vol. 33 (1927), p. 97. 
For $\eta=1$ this is system (29). For $\eta=0$ it is, if we set $u(x, 0)=y(x)$, the differential system

$$
\begin{aligned}
y^{\prime \prime}(x)-\lambda^{2} y(x) & =0, \\
L_{i}(y) & =0,
\end{aligned}
$$

which is equivalent to the integral equation

$$
u(x, 0)=\lambda^{2} \int_{a}^{b} G(x, \xi) u(\xi, 0) d \xi .
$$

We may suppose the parameters $\mu_{i j}, \nu_{i j}$ chosen subject to previous restrictions so that

$$
\left|\begin{array}{cc}
\mu_{12} & \nu_{12} \\
\mu_{22} & \nu_{22}
\end{array}\right| \neq 0, *
$$

and also so that the system (52) has only simple characteristic values. With such a choice the solutions $u_{m}(x, 0)$ of system (52) and the solutions $v_{m}(x, 0)$ of the adjoint system compose a set of functions which is biorthogonal, normalized and closed. We wish to show that for $\eta$ otherwise suitably chosen in the circle $C:|\eta| \leqq 1$ the system (51) still defines a set of solutions $u_{m}(x, \eta)$, and that there exists an associated biorthogonal normalized set $v_{m}(x, \eta)$.

The system (51) is obtained formally from (29) by replacing $f(x, \xi), g(x)$, $\phi_{i}(x)$, and $V_{i}(\xi), i=1,2$, respectively by these functions multiplied by $\eta$. This has the effect of replacing $F(x, \xi)$ in chapter 3 by $\eta F(x, \xi)$, and $\Phi_{i}(x)$, $i=1,2$, by $\Phi_{i}(x, \eta)$, polynomials of the first degreein $\eta$. If the neighborhoods of a finite number of critical points are excluded from the circle $C$ and the remaining region is denoted by $C^{\prime}$, then it can be shown that for $\eta$ in $C^{\prime}$ the following facts obtain. $\dagger$

(a) The function $\eta F(x, \xi)$ possesses a reciprocal $E(x, \xi, \eta)$ which is analytic in $\eta$. Hence by Theorem 4 the system (51) is equivalent to an integral equation

$$
u(x, \eta)=\lambda^{2} \int_{a}^{b} K(x, \xi, \eta) u(\xi, \eta) d \xi
$$

in which the kernel is analytic in $\eta$.

* This is the condition that system (52) be regular. Cf. Birkhoff, Boundary value and expansion problems, these Transactions, vol. 9 (1908), p. 383.

† Paper 1, \$39. 
(b) The integral equation associated with (54) is equivalent to an integrodifferential system of the type considered in the foregoing chapters.

(c) $k_{13}(\eta) k_{24}(\eta)-k_{14}(\eta) k_{23}(\eta) \neq 0$,

where $k_{j i}(\eta)$ is given by formulas (46) etc. with the formal introduction of $\eta$ as noted above.

Hence by chapters 6 and 7 the characteristic values $\lambda_{m}(\eta)$ exist and for $m$ sufficiently large the solutions of (51) are given by formulas (50a), the functions $H(x)$ and $H(x, m)$ being replaced by functions $H(x, \eta)$ and $H(x, m, \eta)$, which are continuous in $x$ and $\eta$.

Since the asserted facts have been established for the system (29) the region $C^{\prime}$ may be chosen to include the value $\eta=1$. Moreover, since

$$
k_{18}(0) k_{24}(0)-k_{14}(0) k_{23}(0)=\mu_{12} \nu_{22}-\mu_{22} \nu_{12} \text {, }
$$

from formulas (46) etc. it follows from (52) that $\eta=0$ may also be included in $C^{\prime}$.

29. The application of the theorem of $\$ 27$. Let $\Gamma$ represent now any closed curve connecting the points $\eta=0$ and $\eta=1$ and lying entirely within the region $C^{\prime}$, and let the values of $\eta$ hereafter considered be restricted to this curve $\Gamma$. Using the symbol $\delta h(x)$ when $h(x, \eta)$ is any function of $x$ and $\eta$ to designate the difference in the value of $h$ for $\eta=\bar{\eta}$ and $\eta=\eta$, i.e.

$$
h(x, \bar{\eta})-h(x, \eta)=\delta h(x),
$$

we obtain from the formulas (50a) with $\eta$ introduced as noted under (c) above the relation

$$
\begin{aligned}
& \sum_{m=M}^{\infty}\left[u_{m}(x, \bar{\eta})-u_{m}(x, \eta)\right] v_{m}(y, \bar{\eta})=\delta H(x) \sum_{m=M}^{\infty} \frac{\sin \frac{m \pi(b-x)}{b-a} \cos \frac{m \pi(b-y)}{b-a}}{m} \\
& +\delta H(x) \sum_{m=M}^{\infty} \frac{\sin \frac{m \pi(x-a)}{b-a} \cos \frac{m \pi(b-y)}{b-a}}{m} \\
& +\sum_{m=M}^{\infty} \frac{H(y, m) \delta H(x, m)}{m^{2}} .
\end{aligned}
$$

Of the series occurring here on the right the last converges uniformly to a value which becomes uniformly small with $|\bar{\eta}-\eta|$. Each of the remaining series may be transformed into a pair of series of the form $\sum_{m=M}^{\infty}(\sin m \pi z) / m$. Such series are known to converge everywhere uniformly save in the immediate vicinity of $z=0, \pm 2, \pm 4, \cdots$, and the sum of any number of terms 
is uniformly bounded. It follows* that the right hand member of (55) converges in the manner demanded by the theorem of $\$ 29$, provided that $|\bar{\eta}-\eta|<\delta$, where $\delta$ is a positive constant chosen sufficiently small. Since the set $\left\{u_{m}(x, 0)\right\}, m=0,1,2, \cdots$, is known to be closed, the sub-set for $m=M, M+1, \cdots$, involves an $M$-fold lack of closure. It follows, therefore, first, that the lack of closure of the set $\left\{u_{m}(x, \eta)\right\}, m=M, M+1, \cdots$, for $|\eta|<\delta$ is at most $M$-fold, and subsequently, after a finite number of repeated applications of the theorem, that the set $\left\{u_{m}(x, 1)\right\}, m=M, M+1, \cdots$, involves at most an $M$-fold lack of closure. Clearly the same deduction for the set $\left\{v_{m}(x, 1)\right\}$ is possible.

We observe now that under the conditions (vii) and (vii) the kernel of the normalized equation cannot be orthogonal to any continuous function. $\dagger$ If we assume also that

(ix) all characteristic values are simple,

then the set of solutions is biorthogonal, and since a sub-set of the characteristic functions involves only a finite lack of closure the entire set is closed. $\ddagger$ Hence the sets $\left\{u_{m}(x, 1)\right\}$ and $\left\{v_{m}(x, 1)\right\}$ are closed and we have the following theorem.

THEOREM 12. If the kernel of the normalized integral equation satisfies the conditions (va), (vi), (vii), (viii), and (ix) and the kernel of the associated equation satisfies the condition (vii), then the sets of characteristic functions $u_{m}(x)$ and $v_{m}(x)$ are closed.

30. Other characteristic values and functions. It is conceivable that the methods employed may not lead to the detection of all existent characteristic values and functions. That this is not the case, however, may be easily shown with the use of Theorem 12.§

\section{Chapter 9. The expansion of an arbitrary function}

31. The equi-convergence property. We consider now in connection with the given integral equation the ordinary differential system (52) with solutions $y_{m}(x)$ and the adjoint system with solutions $z_{m}(x)$. The function $\phi(x, \xi, p)$ we define by the relation

$$
\phi(x, \xi, p)=\sum_{m=0}^{p}\left[u_{m}(x) v_{m}(\xi)-y_{m}(x) z_{m}(\xi)\right] .
$$

- See paper $1, \$ 41$.

$\dagger$ See the discussion in $\$ 10$.

$\ddagger$ Langer, Bulletin of the American Mathematical Society, loc. cit.

8 See paper 1, chapter 11 . 
Inasmuch as the functions $u_{m}(x, \eta), v_{m}(x, \eta)$ become $y_{m}(x), z_{m}(x)$ for $\eta=0$, and $u_{m}(x), v_{m}(x)$ for $\eta=1$, we obtain from formulas (50a) for $M$ a fixed number sufficiently large and $p>M$

$$
\phi(x, \xi, p)=\phi(x, \xi, M)+\sum_{j=1}^{4} \psi_{j}(x, \xi, p)+\sum_{m=M+1}^{p} \frac{B(x, \xi, m)}{m^{2}},
$$

where each of the functions $\psi_{i}$ is of the form

$$
\psi_{i}(x, \xi, p)=H(x, \xi) \sum_{m=M+1}^{p} \frac{\sin m \pi \theta_{i} \cos m \pi \omega_{i}}{m},
$$

and $\theta_{i}, \omega_{i}$ are linear functions of $x$ or $\xi$. As previously observed in the discussion of formula (55) the sums in (57) can each be written as a pair of sums of the form $\sum(\sin m \pi z) / m$. It follows from the properties of series of this type as already noted, and from formulas (56a) and (56), that

$$
|\phi(x, \xi, p)|<A \quad \text { (a constant), }
$$

and that for any choice of the points $\alpha$ and $\beta$ on the interval $(a, b)$

$$
\lim _{p \rightarrow \infty} \int_{\alpha}^{\beta} \phi(x, \xi, p) d \xi=\Phi(x),
$$

the convergence being uniform and $\Phi(x)$ therefore being continuous on $(a, b)$.

To evaluate $\Phi(x)$ we denote by $f(x)$ the function defined as follows:

$$
f(x)=\left\{\begin{array}{l}
1 \text { for } \alpha \leqq x \leqq \beta, \\
0 \text { for } a \leqq x<\alpha \text { and } \beta<x \leqq b .
\end{array}\right.
$$

Since the system (52) is regular by (53) and has only simple characteristic values we have

$$
\sum_{m=0}^{\infty} \int_{a}^{b} f(\xi) z_{m}(\xi) d \xi \cdot y_{m}(x)=\bar{f}(x), *
$$

where $\bar{f}(x)$ is bounded and differs from $f(x)$ at most in the points $\alpha, \beta, a, b$. Except in the neighborhoods of these points moreover, the convergence is uniform as may be seen by using formulas (50a). But

whence

$$
\int_{a}^{b} f(\xi) \phi(x, \xi, p) d \xi=\int_{\alpha}^{\beta} \phi(x, \xi, p) d \xi
$$

$$
\Phi(x)=\sum_{m=0}^{\infty} \int_{a}^{b} f(\xi) v_{m}(\xi) d \xi \cdot u_{m}(x)-\bar{f}(x) .
$$

\footnotetext{
* Birkhoff, these Transactions, vol. 9 (1908), p. 390.
} 
Multiplying this relation by $v_{l}(x)$ and integrating as we may because of the uniformity noted above, we obtain, since the system $\left\{u_{l}(x), v_{l}(x)\right\}$ is normalized and biorthogonal, the result

$$
\int_{a}^{b} \Phi(x) v_{l}(x) d x=0 \text { for all } l .
$$

Since the set $v_{l}(x)$ is closed it follows that $\Phi(x) \equiv 0$, namely that

$$
\lim _{p \rightarrow \infty} \int_{\alpha}^{\beta} \phi(x, \xi, p) d \xi=0
$$

uniformly in $x$ and for all $\alpha$ and $\beta$ on $(a, b)$.

The relations (58) and (59) are the hypotheses of a theorem by Lebesgue* which asserts that

$$
\lim _{p \rightarrow \infty} \int_{a}^{b} f(\xi) \phi(x, \xi, p) d \xi=0
$$

uniformly for any function $f(x)$ which is summable over $(a, b)$. We have, therefore, the following theorems.

ThEOREM 13. If $f(x)$ is any function which is summable over the interval $(a, b)$, and if

$$
\begin{aligned}
& F_{1 p}(x)=\sum_{m=0}^{p} \int_{a}^{b} f(\xi) v_{m}(\xi) d \xi \cdot u_{m}(x), \\
& F_{2 p}(x)=\sum_{m=0}^{p} \int_{a}^{b} f(\xi) z_{m}(\xi) d \xi \cdot y_{m}(x),
\end{aligned}
$$

where $u_{m}(x), v_{m}(x)$ are respectively the normalized solutions of the integral equation in normal form and the associated equation and $y_{m}(x), z_{m}(x)$ are respectively the normalized solutions of a related differential system (52) and its adjoint system, then

$$
\lim _{p \rightarrow \infty}\left[F_{1 p}(x)-F_{2 p}(x)\right]=0 \text { uniformly. }
$$

A more explicit but less inclusive formulation may be had by drawing on the expansion theorem of the related differential system. $\dagger$ Thus we may state

THEOREM 13a. If $f(x)$ is a function which on the interval $(a, b)$ consists of a finite number of pieces, each real, continuous, and with a continuous derivative then the expansion

* Annales de la Faculté des Sciences de Toulouse, vol. 23 ((3), vol. 1) (1909), p. 52 and p. 68.

$\dagger$ Birkhoff, loc. cit. 


$\begin{array}{ll} & \frac{1}{2}[f(x+)+f(x-)] \quad \text { for } a<x<b, \\ & a_{1} f(a+)+a_{2} f(b-) \text { for } x=a,\end{array}$

and to

$$
\sum_{m=0}^{\infty} \int_{a}^{b} f(\xi) v_{m}(\xi) d \xi \cdot u_{m}(x)
$$

$$
b_{1} f(a+)+b_{2} f(b-) \text { for } x=b,
$$

where $a_{1}, a_{2}, b_{1}, b_{2}$ are constants independent of $f(x)$.

Chapter 10. The asymptotic forms under alternative hypotheses

32. The characteristic values. The form of the characteristic equation (45a) and hence also the forms for the characteristic values and functions above were obtained as a result of the hypothesis (viii). If this hypothesis is not met, the character of the solutions is in general quite different. This will be illustrated in the following discussion.

Let us assume that the integral equation in hand is such that

$$
\begin{gathered}
k_{13} k_{24}-k_{14} k_{23}=0, \\
C=\left|\begin{array}{ll}
k_{11} & k_{14} \\
k_{21} & k_{24}
\end{array}\right|+\left|\begin{array}{ll}
k_{12} & k_{13} \\
k_{22} & k_{23}
\end{array}\right| \neq 0 .
\end{gathered}
$$

If the forms (43a) are substituted in (45) then, certain terms in the expansion of the resulting determinant vanish because of the first of conditions ( $x$ ). Substituting in the remaining terms the forms (40a) we find that the characteristic equation is of the type

$$
[C+\epsilon(\lambda)] e^{\lambda(b-a)}-2[B+\epsilon(\lambda)]+[C+\epsilon(\lambda)] e^{-\lambda(b-a)}=0,
$$

where

$$
B=\left|\begin{array}{ll}
k_{13} & k_{11} \\
k_{23} & k_{21}
\end{array}\right|+\left|\begin{array}{ll}
k_{14} & k_{12} \\
k_{24} & k_{22}
\end{array}\right| \text {. }
$$

If $Q$ and $1 / Q$ denote the roots of the quadratic

$$
z^{2}-2 \frac{B}{C} z+1=0
$$

it can be shown by the method of $\$ 22$ that for $|\lambda|>N$ the equation (60) admits of just one root in the neighborhood of each of the points

$$
\begin{aligned}
\rho_{m} & =\frac{1}{b-a}[2 m \pi i+\log Q] \quad(m= \pm M, \pm(M+1), \cdots), \\
\rho_{m 1} & =\frac{1}{b-a}[2 m \pi i-\log Q] .
\end{aligned}
$$


We shall denote these roots respectively by $\lambda_{m}$ and $\lambda_{m_{1}}$. Observing now that on the one hand $-\lambda_{m}$ must be a characteristic value since the equation (7) involves $\lambda$ only to an even power, while on the other hand $\rho_{m_{1}}=-\rho_{-m}$, it becomes clear that $\lambda_{m_{1}}=-\lambda_{-m}$. Hence we have the following theorem:

ThEOREM 14. Under the hypothesis (v), (vi), and (x), and either (vii) or (vii), the normalized integral equation (7) admits of infinitely many characteristic values. For $|m|$ sufficiently large these values are given by the formulas

$$
\begin{aligned}
\lambda_{m} & =\frac{1}{b-a}[2 m \pi i+\log Q]+\epsilon_{m} \quad(m= \pm M, \pm(M+1), \cdots), \\
\lambda_{m_{1}} & =-\lambda_{m} .
\end{aligned}
$$

33. The characteristic functions. It is not possible in this case to choose the constants $h_{1}$ and $h_{2}$ to satisfy (49). Instead we may choose them so that

$$
h_{1} k_{13}+h_{2} k_{23}=0, h_{1} k_{14}+h_{2} k_{24}=0 .
$$

With this choice we obtain in place of (48) the relation

$$
c_{1}: c_{2}=-U_{2}\left(\lambda_{m}\right): U_{1}\left(\lambda_{m}\right),
$$

where

$$
\begin{aligned}
U_{i}(\lambda)=\left(h_{1} k_{11}+h_{2} k_{21}\right) u_{i}(a)+\left(h_{1} k_{12}+h_{2} k_{22}\right) u_{i}(b) \\
-\int_{a}^{b}\left[h_{1} \Omega_{1}(\xi)+h_{2} \Omega_{2}(\xi)\right] u_{i}(\xi) d \xi .
\end{aligned}
$$

Substituting in this the forms (40a) and observing that

we find

$$
e^{\lambda m(b-a)}=Q+\epsilon(m)
$$

$$
c_{1}: c_{2}=-\left(k_{1}+\epsilon\right):\left(k_{2}+\epsilon\right) \text {, }
$$

where

$$
\begin{aligned}
& k_{1}=\left(h_{1} k_{11}+h_{2} k_{21}\right)+\left(h_{1} k_{12}+h_{2} k_{22}\right) Q^{-1}, \\
& k_{2}=\left(h_{1} k_{11}+h_{2} k_{21}\right)+\left(h_{1} k_{12}+h_{2} k_{22}\right) Q .
\end{aligned}
$$

Under condition ( $\mathrm{x}$ ) these two constants cannot vanish simultaneously. Inasmuch as

$$
e^{\lambda_{m} x}=e^{\rho_{m} x}+\epsilon(m, x),
$$

we obtain from (40a) and (42) a form for the solutions as given in the following theorem: 
THEOREM 15. Under the hypotheses (v), (vi), (vii) and (x) the characteristic functions of the normalized integral equation are given for $|m|$ sufficiently large by an expression of the form

$$
u_{m}(x)=k_{1} e^{\rho_{m}(x-a)}-k_{2} e^{-\rho_{m}(x-a)}+\epsilon(x, m),
$$

the coefficients $k_{1}$ and $k_{2}$ being constants, not both zero.*

34. A further case. It is clear that the method employed will in fact be applicable whenever the characteristic equation is of the form

$$
\left[c_{1}+\epsilon(\lambda)\right] e^{\lambda(b-a)}+\left[c_{2}+\epsilon(\lambda)\right]+\left[c_{3}+\epsilon(\lambda)\right] e^{-\lambda(b-a)}=0 \text {, and } c_{1} c_{3} \neq 0 \text {. }
$$

The following is an example in which this holds although conditions (viii) and $(\mathrm{x})$ both fail to be fulfilled.

Let $K(x, \xi)$ be any kernel satisfying condition (vi) and the further condition

$$
K(x, a) \equiv K(x, b) \equiv K(a, \xi) \equiv K(b, \xi) \equiv 0 .
$$

We shall choose the parameters $\mu_{i j}, \nu_{i j}$ so that $\mu_{11}=\nu_{21}=1$, while the others all vanish. This choice is contrary to (13) but the discussion is nevertheless found to be valid. We obtain then $K(x, \xi) \equiv K(x, \xi), \theta_{i}(x) \equiv 0, \phi_{i}(x) \equiv 0$, $V_{i}(\xi) \equiv 0, i=1,2$, and $k_{11}=k_{21}=1$, while the other quantities $k_{i j}$ are all zero. The equations (43) reduce to $\omega_{1 j}=u_{j}(a), \omega_{2 j}=u_{j}(b)$, and the characteristic equation is found to be of the form (45a). Hence the characteristic values are given by $(45 b)$.

In determining the characteristic functions we may choose $h_{1}=0, h_{2}=1$ to satisfy (63). This leads to

$$
c_{1}: c_{2}=-\left(e^{-\lambda_{m} b}+\epsilon\right):\left(e^{\lambda_{m} b}+\epsilon\right),
$$

and with these values we find as the normalized characteristic functions

$$
u_{m}(x)=\left(\frac{2}{b-a}\right)^{1 / 2} \sin \frac{m \pi(b-x)}{b-a}+\epsilon(x, m) .
$$

It is clear that throughout this chapter more precise forms for the characteristic values and functions are obtainable by the methods used precisely as in $\$ 23$ and $\$ 26$.

* If the kernel of the equation considered in paper 1 is written $K_{1}(x, \xi)$, the iterated kernel $K(x, \xi)=\int_{a}^{b} K_{1}(x, t) K_{1}(t, \xi) d t$ is readily found to satisfy the hypotheses made concerning the equation discussed in the present paper. Hence the characteristic values and solutions obtained in paper 1 must be included among those derived here. A comparison of the results shows them to be included here among the equations satisfying condition $(x)$.

BROWN UNIVERSITY, Providence, R. I. 